\title{
On transmission performance of OFDM-based schemes using MMSE-FDE in a frequency- selective fading channel
}

\author{
Haris Gacanin ${ }^{1 *}$ and Fumiyuki Adachi ${ }^{2}$
}

\begin{abstract}
There has been greatly increasing interest in orthogonal frequency division multiplexing (OFDM) for broadband wireless transmission due to its robustness against multipath fading. However, OFDM signals have high peak-toaverage power ratio (PAPR), and thus, a power amplifier must be operated with a large input power backoff (IBO). Recently, OFDM combined with time division multiplexing (OFDM/TDM) using minimum mean square errorfrequency domain equalization (MMSE-FDE) has been presented to reduce the PAPR, while improving the bit error rate (BER) performance of conventional OFDM. In this article, by extensive computer simulation, we present a comprehensive performance comparison of OFDM-based schemes in a nonlinear and frequency-selective fading channel. We discuss about the transmission performance of OFDM-based schemes with respect to the transmit peak-power, the achievable capacity, the BER performance, and the signal bandwidth. Our results show that OFDM/TDM using MMSE-FDE achieves a lower peak-power and capacity than conventional OFDM, which means significant reduction of amplifier transmit-power backoff, but with a slight decrease in signal bandwidth occupancy.
\end{abstract}

Keywords: OFDM/TDM, OFDM, capacity, power spectrum density, bit error rate, amplifier power efficiency

\section{Introduction}

In a wireless channel, a signal propagates over a number of different paths that give rise to a frequency-selective fading, which produce severe inter-symbol interference (ISI) and degrades the transmission performance [1]. To solve this problem, intensive research effort on frequency domain channel equalization (FDE) is currently ongoing in two directions: (i) orthogonal frequency division multiplexing (OFDM) [2], and (ii) single carrier (SC)-FDE [3]. To avoid the performance degradation of OFDM due to high PAPR, the high transmit power amplifier (HPA) must be operated with a large input backoff (IBO). Otherwise, the system performance in terms of the bit error rate (BER), channel capacity, throughput, etc., may be degraded. The performance of OFDM system over a nonlinear channel (e.g., HPA or amplitude limiter) has been analyzed in the recent literature [4-6].

\footnotetext{
* Correspondence: harisg@ieee.org

${ }^{1}$ Motive Division, Alcatel-Lucent Bell N.V., Antwerp, Belgium

Full list of author information is available at the end of the article
}

Of late, various approaches to reduce the PAPR of OFDM have been proposed [7-12]. The conventional OFDM and SC-FDE are compared in [13] with respect to their BER performances, PAPR, carrier frequency offset, and computational complexity. In [14], the performance of clipped OFDM is analyzed in terms of the PAPR reduction capability and degradation of the channel capacity. It was shown that the nonlinearity significantly degrades the channel capacity of OFDM due to the high PAPR.

Recently, OFDM combined with time division multiplexing (OFDM/TDM) [15] using minimum mean square error FDE (MMSE-FDE) [16] was presented to reduce the PAPR, while improving the BER performance of conventional OFDM. The PAPR problem, however, cannot be completely eliminated. OFDM/TDM using MMSE-FDE transmits data over $N_{m}\left(=N_{c} / K\right)$ subcarriers, where $N_{c}$ is the number of subcarriers in the conventional OFDM. A natural consequence is that the capacity may decrease due to the reduced number of subcarriers. In particular, as stated in [14], the channel capacity further decreases in a nonlinear channel due to 
the PAPR problem of OFDM. Hence, some additional PAPR reduction technique must be applied.

In [17], we analyzed the theoretical BER performance of amplitude clipped and filtered OFDM/TDM using MMSE-FDE. However, to unveil a potential of OFDM/ TDM using MMSE-FDE, a more detailed transmission performance comparison in terms of transmit peakpower, the channel capacity and the spectrum splatter of OFDM/TDM, and the conventional OFDM is required. To the best of our knowledge, such performance comparison between OFDM/TDM using MMSE-FDE and the conventional OFDM has not been reported.

In this article, we provide a comprehensive performance comparison between OFDM/TDM using MMSEFDE and the conventional OFDM. A trade-off between the transmit peak-power reduction (i.e., IBO reduction), the achievable capacity, the BER performance and the power spectrum efficiency is discussed. We discuss about how, and by how much, OFDM/TDM using MMSE-FDE improves the transmission performance in comparison with conventional OFDM system. Our aim is to show that OFDM/TDM using MMSE-FDE can be used in practical systems to overcome the high PAPR problem of conventional OFDM at the cost of slight decrease in spectrum efficiency. The capacity of OFDM/ TDM using MMSE-FDE is obtained based on the Gaussian assumption of the OFDM/TDM signal amplitude.

The remainder of this article is organized as follows. Section II presents OFDM/TDM using MMSE-FDE system model. The computer simulation results and discussions are presented in Section III. Section IV concludes the article.

\section{System overview}

In this section, we begin with a brief overview of the conventional OFDM and later, OFDM/TDM using MMSE-FDE is presented. The information bit sequence of length $M$ is channel coded with a coding rate $R$ and mapped into the transmit data symbols, corresponding to quadrature phase shift keying (QPSK) modulation scheme. This sequence is divided into blocks $\left\{d_{m}(i) ; i=\right.$ $\left.0 \sim N_{c}-1\right\}, m=0 \sim M / N_{c}-1$, with $E\left[\left|d_{m}(i)\right|^{2}\right]=1$, where $E[\cdot]$ denotes the ensemble average operation. In this study, without loss of generality, we consider a transmission of one block and thus, the block index $m$ is omitted in what follows.

\section{A. Conventional OFDM}

The conventional OFDM system model is illustrated in Figure 1. In the conventional OFDM system, an $N_{c}$ datamodulated symbol sequence $\left\{d(i) ; i=0 \sim N_{c}-1\right\}$ is fed to $\mathrm{JN}_{c}$-point inverse fast Fourier transform (IFFT) to generate an oversampled time-domain OFDM signal with $N_{c}$ subcarriers. Throughout this study, the oversampling

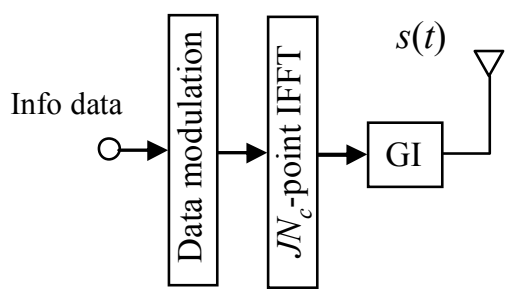

(a) Transmitter

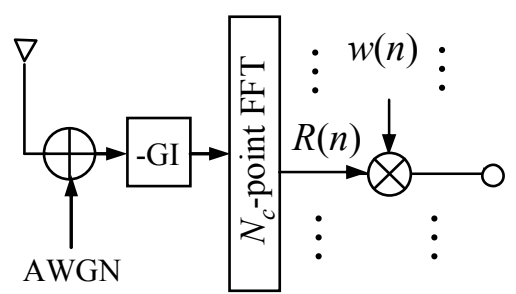

(b) Receiver

Figure 1 Conventional OFDM transmitter/receiver structure.

ratio $J$ is used to approximate the time domain transmit signal with high accuracy. After insertion of guard interval (GI) the signal is fed to pre-linearized HPA (i.e., the signal is clipped and filtered by a soft-limiter model), where linear amplification is achieved until the saturation output power level $P_{s}$ (normalized by the input signal power). We assume that the amplifier saturation level equals the clipping level. Finally, the signal is transmitted over a frequency-selective fading channel.

At the receiver, after removing the GI, the $N_{c}$-point FFT is applied to decompose the received signal into $N_{c}$ subcarriers $\left\{R(n) ; n=0 \sim N_{c}-1\right\}$. The distortion in the channel has the effect of changing the phase and amplitude of each subcarrier, which is corrected by the single tap FDE through multiplication of the received signal $R$ $(n)$ by the equalization weight $w(n)$ [2].

\section{B. OFDM/TDM using MMSE-FDE}

The OFDM/TDM transmission system model is illustrated in Figure 2. In OFDM/TDM the $N_{c}$-subcarrier OFDM signaling interval (i.e., OFDM/TDM frame) is divided into $K$ slots. A date-modulated symbol sequence $\left\{d(i) ; i=0 \sim N_{c}\right.$ $-1\}$ to be transmitted is divided into $K$ subblocks each having $N_{m}\left(=N_{c} / K\right)$ data-modulated symbols. A time and frequency symbol arrangement for conventional OFDM and OFDM/TDM is presented in Figure 3 . The $k$ th subblock $\left\{d^{k}(i) ; i=0 \sim N_{m}-1\right\}$ is transmitted in the kth slot, where $d^{k}(i)=d\left(k N_{m}+i\right)$ for $k=0 \sim K-1$. Then, JN $\mathrm{N}_{m}$-point IFFT is applied to generate the $k$ th slot oversampled timedomain OFDM signal with $N_{m}$ subcarriers as

$$
s^{k}(t)=\sqrt{2 P} \sum_{i=0}^{N_{m}-1} d^{k}(i) \exp \left\{j 2 \pi t \frac{i}{\mathrm{JN}_{m}}\right\}
$$



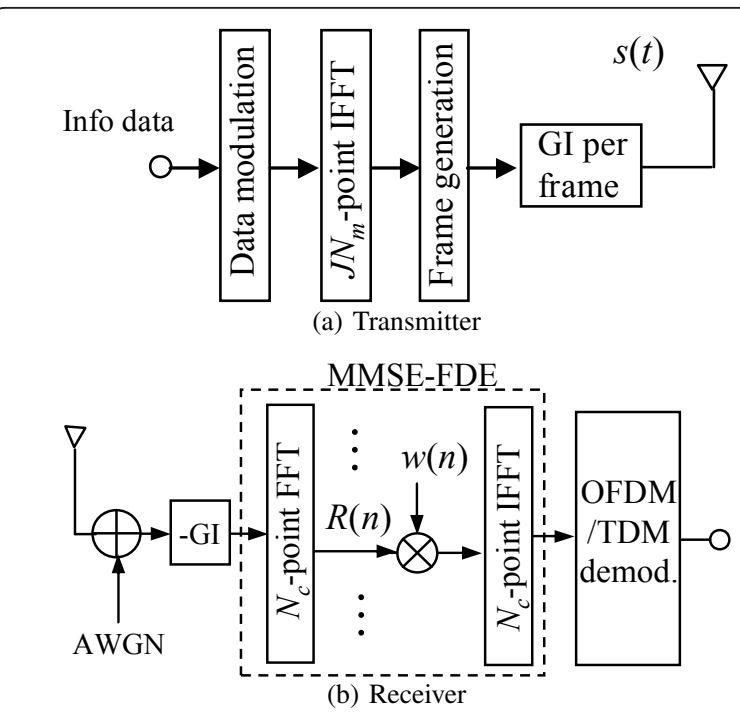

Figure 2 OFDM/TDM transmitter/receiver structure.

for $t=0 \sim N_{m}-1$, where $P=E_{s} / T_{c} N_{m}$ denotes the transmit signal power. $E_{s}$ and $T_{c}$ denote the data-modulated symbol energy and the sampling interval of the IFFT, respectively. The OFDM/TDM signal can be expressed using the equivalent low-pass representation as

$$
s(t)=\sum_{k=0}^{K-1} s^{k}\left(t-k N_{m}\right) u\left(t-k N_{m}\right)
$$

for $t=0 \sim N_{c}-1$, where $u(t)=1(0)$ for $t=0 \sim N_{m}$ 1 (elsewhere). After insertion of the guard interval (GI), the OFDM/TDM signal is fed into pre-linearized HPA as in the case of conventional OFDM and transmitted over a frequency-selective fading channel.

The OFDM/TDM signal propagates through the channel with a discrete-time channel impulse response $h(\tau)$ given as

$$
h(\tau)=\sum_{l=0}^{L-1} h_{l} \delta\left(\tau-\tau_{l}\right),
$$

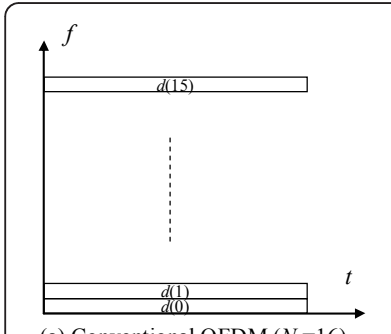

(a) Conventional OFDM $\left(N_{c}=16\right)$

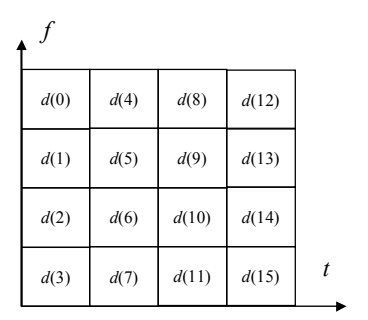

(b) OFDM/TDM $\left(N_{c}=16 ; N_{m}=4, K=4\right.$
Figure 3 Time and frequency data arrangement where $h_{l}$ and $\tau_{l}$ are the path gain and the time delay, respectively, of the $l$ th path having the sample-spaced exponential power-delay profile with channel decay factor $\beta$ (i.e., $E\left[\left|h_{g, l}\right|^{2}\right]=\frac{1-\beta}{1-\beta^{L}} \beta^{l}$ ). We assume that the maximum time delay of the channel is less than the GI length.

At the receiver, $N_{c}$-point FFT is applied over the entire OFDM/TDM frame [16] to decompose the received signal into $N_{c}$ frequency components represented by $\left\{R(n) ; n=0 \sim N_{c}-1\right\}$. One-tap MMSE-FDE [3] is applied to $R(n)$ as

$$
\hat{R}(n)=R(n) w(n)
$$

where $w(n)$ is the equalization weight given by [16]

$$
w(n)=\frac{H^{*}(n)}{|H(n)|^{2}+\left(\frac{E_{s}}{N_{0}}\right)^{-1}}
$$

where $H(n)$ and $N_{0}$ denote the Fourier transform of the channel impulse response and the single-sided additive white Gaussian noise (AWGN) power spectrum density (PSD), respectively.

The time-domain OFDM/TDM signal is recovered by applying $N_{c}$-point IFFT to $\left\{\hat{R}(n) ; n=0 \sim N_{c}-1\right\}$ and then, the OFDM demodulation is carried out using $N_{m^{-}}$ point FFT to obtain decision variables $\left\{\hat{d}^{k}(i) ; \quad i=0 \sim N_{m}-1\right\}[16]$. For channel decoding, the log-likelihood ratios (LLRs) are computed before decoding [18].

We note here that OFDM/TDM using MMSE-FDE for $K=1$ (i.e., $N_{m}=N_{c}$ ) reduces to the conventional OFDM system with $N_{c}=256$ subcarriers.

\section{Performance analysis}

We first develop a mathematical model for PAPR distribution of OFDM/TDM signal and then, we develop the expression for the capacity of OFDM/TDM using MMSE-FDE.

\section{A. PAPR of OFDM/TDM}

The baseband oversampled OFDM/TDM signal given by (2) is considered. The PAPR of the observed OFDM/ TDM frame is defined as the ratio of the peak power to the ensemble average power and can be expressed as

$$
\operatorname{PAPR}=\frac{\max \left\{|s(t)|^{2}\right\}_{\mathrm{t}=0 \sim \mathrm{N} \mathrm{N}_{c}-1}}{E\left\{|s(t)|^{2}\right\}} .
$$

The expression for PAPR distribution of OFDM/TDM is derived based on assumption that $\mathrm{JN}_{m}$-point IFFT size is large enough so that real and imaginary part of 
the $k$ th time slot OFDM signal $s^{k}(t)$, for $t=0 \sim \mathrm{JN}_{m}-1$, are samples of zero-mean statistically independent Gaussian process with unit variance. Hence, the amplitudes $\left\{r(t)\left(=\left|s^{k}(t)\right|\right) ; t=0 \sim \mathrm{JN}_{m}-1\right\}$ are independentand-identically distributed (i.i.d.) Rayleigh random variables [1].

Cumulative distribution function (cdf) $F\left(\lambda_{k}\right)$ of the PAPR $\lambda_{k}$ for the $k$ th slot is given by

$$
F\left(\lambda_{k}\right)=\left[1-\exp \left(-\lambda_{k}\right)\right]^{\mathrm{JN} m} .
$$

We assume that the block data-modulated symbols $\left\{d^{k}\right.$ (i); $\left.i=0 \sim N_{m}-1\right\}$ and $k=0 \sim K-1$ are statistically independent, so that the OFDM/TDM signal is generated from $K$ statistically independent OFDM signals. Hence, the PAPR probability of OFDM/TDM is given by

$$
F_{\text {OFDM } / \text { TDM }}(\lambda)=\left\{1-\left[1-\exp (-\lambda)^{\mathrm{JN}}\right]\right\}^{K} .
$$

It can be seen from (8) that the PAPR of OFDM/ TDM decreases as $K$ increases. For $K=1$, the above expression collapses to the PAPR expression for the conventional OFDM. The above PAPR probability expression given by (8) together with computer simulation results is evaluated in the next section.

\section{B. Channel capacity of OFDM/TDM using MMSE-FDE}

From here on, we analyze capacity of the OFDM/TDM using MMSE-FDE based on the assumption that nonlinear distortion caused by power amplifier is Gaussian. We assume perfect channel knowledge.

Using the Bussgang theorem [5,6], the received OFDM/TDM signal can be expressed as

$$
R(n)=\alpha S(n) H(n)+\alpha I(n)+S_{c}(n) H(n)+N(n) .
$$

where $S(n), H(n), I(n), S_{c}(n)$, and $N(n)$ denote the Fourier transform of transmitted OFDM/TDM signal, the channel gain, the inter-slot interference (ISI), the nonlinear distortion, and zero mean AWGN process, respectively, having single-sided power spectrum density $N_{0} . \alpha$ denotes the attenuation constant that can be well approximated as $\alpha=1-\exp \left(-P_{s}^{2}\right)+\frac{\sqrt{\pi} P_{s}}{2} \operatorname{erfc}\left(P_{s}\right)[4-6]$, where $P_{s}$ is the HPA power saturation level (normalized by the input average signal power), and $\operatorname{erfc}[x]=\frac{2}{\sqrt{\pi}} \int_{x}^{\infty} \exp \left(-t^{2}\right) d t$ is the complementary error function.

After MMSE-FDE, the time-domain OFDM/TDM signal is recovered by applying $N_{c}$-point IFFT to $\left\{\hat{R}(n) ; n=0 \sim N_{c}-1\right\}$ and then, OFDM demodulation is carried out by $N_{m}$-point FFT to obtain decision variables:

$$
\hat{d}^{k}(i)=\sqrt{\frac{2 E_{s}}{T_{c} N_{m}}} \alpha d^{k}(i)\left[\frac{1}{N_{c}} \sum_{n=0}^{N_{c}-1} \hat{H}(n)\right]+\mu^{k}(i)
$$

with $\hat{H}(n)=H(n) w(n)$. In the above expression, $\mu^{k}(i)$ denotes the $k$ th slot composite noise (i.e., the sum of nonlinear component, AWGN, and residual ISI after FDE). We approximate $\mu^{k}(i)$ as a zero-mean complexvalued Gaussian process and that $\mu^{k}(i)$ is uncorrelated with $d^{k}(i)$. Thus, the variance of $\mu^{k}(i)$ can be computed as

$$
\begin{aligned}
2 \sigma^{2}= & \frac{2 \alpha^{2} E_{s}}{T_{c} N_{c}} \sum_{n=0}^{N_{c}-1}\left|\hat{H}(n)-\frac{1}{N_{c}} \sum_{m=0}^{N_{c}-1} \hat{H}(m)\right|^{2}|\Psi(n)|^{2} \\
& +\frac{2 E_{s} N_{m}}{T_{c} N_{c}} \sum_{n=0}^{N_{c}-1}\left[1-\exp \left(-P_{s}^{2}\right)-\alpha^{2}\right]|\hat{H}(n)|^{2}|\Psi(n)|^{2} \\
& +\frac{2 N_{0}}{T_{c} N_{c}} \sum_{n=0}^{N_{c}-1}|w(n)|^{2}|\Psi(n)|^{2}
\end{aligned}
$$

where

$$
\Psi(n)=\frac{1}{N_{m}} \frac{\sin \left\{\pi N_{m} \frac{n-K i}{N_{c}}\right\}}{\sin \left\{\pi \frac{n-K_{i}}{N_{c}}\right\}} \times \exp \left\{j \pi\left[(2 k+1) N_{m}-1\right] \frac{n-K i}{N_{c}}\right\} .
$$

We note here that the first term in (11) denotes the residual ISI, and it is omitted in the case of the conventional OFDM.

For the given $P_{s}$ and $E_{s} / N_{0}$, the ergodic channel capacity $C\left[E_{s} / N_{0}, P_{s}\right]$ in bps $/ \mathrm{Hz}$ over a Rayleigh channel can be computed as [1]:

$$
\begin{aligned}
C\left[E_{s} / N_{0}, P_{s}\right] & =E\left[C\left(\frac{E_{s}}{N_{0}}, P_{s,}\{H(n)\}\right)\right] \\
& =\int_{0}^{\infty} \cdots \int_{0}^{\infty} C\left[\frac{E_{s}}{N_{0}}, P_{s,}\{H(n)\}\right] \wp[\{H(n)\}] \times \prod_{n} \mathrm{~d} H(n),
\end{aligned}
$$

where $C\left(E_{s} / N_{0},\{H(n)\}\right)$ and $\wp[\{H(n)\}]$ denote the conditional channel capacity given by $[1]$ :

$$
C\left[\frac{E_{s}}{N_{0}}, P_{s,}\{H(n)\}\right]=\frac{1}{N_{c}} \sum_{n=0}^{N_{c}-1} \log _{2}\left[1+\gamma\left(\frac{E_{s}}{N_{0}}, P_{s,},\{H(n)\}\right)\right] .
$$

and the joint probability density function of $\{H(n) ; n=$ $\left.0 \sim N_{c}-1\right\}$, respectively. A closed or convenient expression for numerical calculation has not been found for integral in (13), and thus, we resort to a different approach. The signal-to-noise plus interference-and-distortion ratio $\gamma(\cdot)$ of OFDM/TDM using MMSE-FDE is first computed using (10) as

$$
\gamma\left(\frac{E_{s}}{N_{0}}, P_{s},\{H(n)\}\right)=\frac{2 \alpha^{2} \frac{E_{s}}{T_{c} N_{m}}\left|\frac{1}{N_{c}} \sum_{n=0}^{N_{c}-1} \hat{H}(n)\right|^{2}}{2 \sigma^{2}} .
$$


Using (15), we can write (13) as

$$
C\left[\frac{E_{s}}{N_{0}}, P_{s}\right]=\frac{1}{N_{c}} \int_{0}^{\infty} \cdots \int_{0}^{\infty} \sum_{n=0}^{N_{c}-1} \log _{2}\left[1+\gamma\left(\frac{E_{s}}{N_{0}},\{H(n)\}\right)\right] \times \wp[\{H(n)\}] \prod_{n} \mathrm{~d} H(n) .
$$

The evaluation of the ergodic capacity is done by Monte Carlo numerical-computation method as follows. A set of path gains $\left\{h_{l} ; l=0 \sim L-1\right\}$ is generated using (3) to obtain channel gains $\left\{H(n) ; n=0 \sim N_{c}-1\right\}$. Then, the capacity given by (16) is computed using (15) for the given set of channel gains $\{H(n)\}$ as a function of the $E_{s} / N_{0}$ and the normalized saturation level $P_{s}$ of the power amplifier. This is repeated a sufficient number of times to obtain the average capacity.

\section{Iv. Numerical evaluation and discussions}

We assume an OFDM/TDM frame size of $N_{c}=256$ samples, GI length of $N_{g}=32$ samples, and ideal coherent quadrature phase shift keying (QPSK) data modulation/demodulation. As the propagation channel, we assume an $L=16$-path block Rayleigh fading channel having the exponential power-delay profile with channel decay factor $\beta$. It is assumed that the maximum time delay of the channel is less than the GI length. The information bit sequence length is taken to be $M=$ 1024 bits. A $(2048,1024)$ low-density parity check (LDPC) encoder [19] is assumed with code rate $R$, and sum product algorithm (SPA) decoder having column weight $=1$, and row weight $=8$. A rate $R=1 / 3$ turbo encoder with constraint length 4 and $(13,15)$ recursive systematic convolutional (RSC) component encoders is applied, while the parity bit sequences are punctured to obtain coding rate of $1 / 2$. The turbo coded bit sequence is interleaved before data modulation. A block interleaver used as channel interleaver in the simulation is of size $2 \mathrm{a}$ and $2 \mathrm{~b}$ block interleaver, where $\mathrm{a}$ and $\mathrm{b}$ are the maximum allowable integers for a given sequence size so that we can obtain an interleaver as close as possible to a square one. The internal interleaver for turbo coding is S-random $\left(S=N^{\frac{1}{2}}\right)$ interleaver. Log-MAP decoding with eight iterations is carried out at the receiver.

\section{A. Bit error rate issue}

The BER performance with and without channel coding as a function of the average signal energy per bit-toAWGN power spectrum density ratio $E_{b} / N_{0}=0.5 \times R \times$ $\left(E_{s} / N_{0}\right) \times\left(1+N_{g} / N_{c}\right)$ is illustrated in Figure 4 . In our simulation, we consider turbo and LDPC channel encoders with rate $R=1 / 2$. As seen from Figure 4a, the coded BER of conventional OFDM $(K=1)$ is better than OFDM/TDM with $K=16$ (64) (i.e., 1.4 (0.15) dB lower $E_{b} / N_{0}$ is required to achieve BER $\left.=10^{-4}\right)$. Unlike

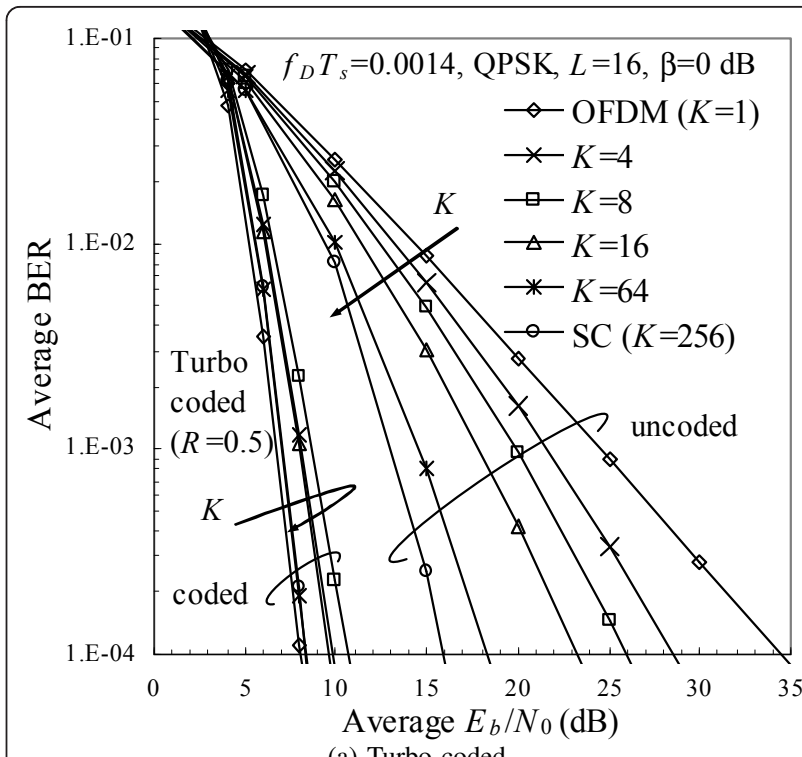

(a) Turbo coded

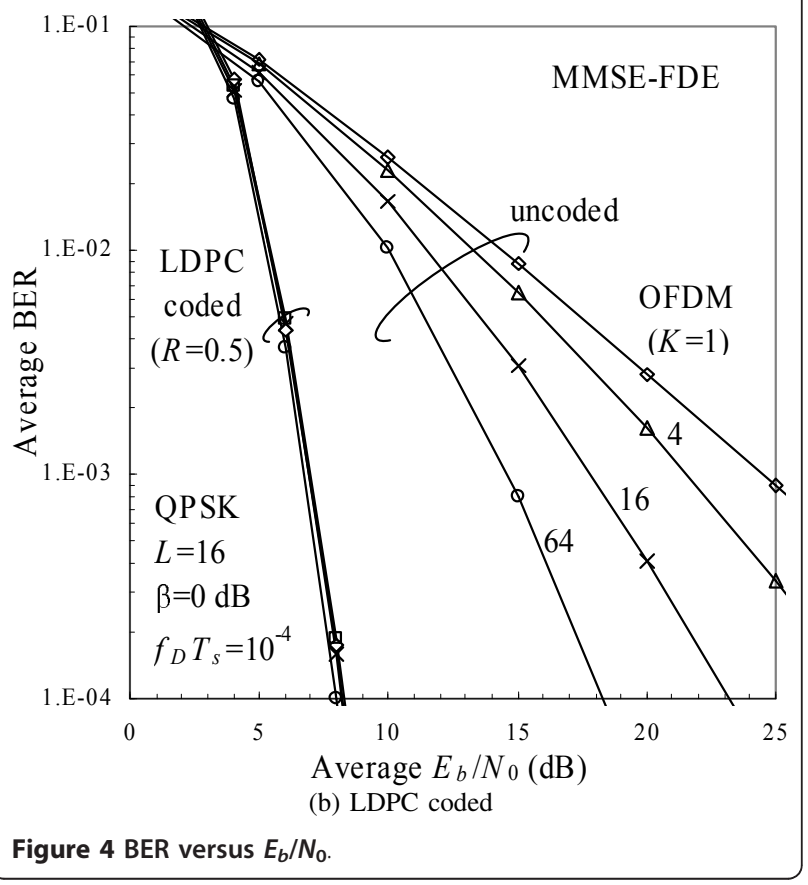

uncoded case where the BER decreases as $K$ increases, with turbo coding, a trade-off is present among frequency diversity gain, coding gain due to better frequency interleaving effect, and orthogonality distortion between consecutive slots within OFDM/TDM frame; for higher (lower) $K$, the coding gain is lower (higher) due to the reduced frequency-interleaving effect, while higher (lower) frequency diversity gain is obtained. Consequently, for turbo-coded case, the appropriate parameter $K$ may be chosen to achieve the same BER as conventional OFDM while still giving the lower PAPR. It can be seen from the Figure $4 \mathrm{~b}$ that the LDPC-coded 
BER performance is almost the same irrespective of the designed parameter $K$.

Figure 5 illustrates the average BER performance of OFDM/TDM with MMSE-FDE as a function of the amplifier's saturation power level $P_{s}$ normalized by the input signal power for $E_{b} / N_{0}=30 \mathrm{~dB}$ with $K$ as a parameter. The figure shows that OFDM/TDM can be used to reduce the required $\mathrm{IBO}$, while achieving the better BER than the conventional OFDM. For example, if the required $\mathrm{BER}=10^{-3}$, then the conventional OFDM $(K=$ 1) cannot achieve this performance irrespective of $P_{s}$. Hence, to achieve BER $=10^{-3}$ with reduced IBO, we can use OFDM/TDM. When $K$ increases from 16 to 32, the HPA power saturation level $P_{s}$ can be reduced from 7 to $1 \mathrm{~dB}$ for $\mathrm{BER}=10^{-3}$, respectively. Note that $K=64 \mathrm{can}$ achieve $\mathrm{BER}=10^{-3}$ irrespective of $P_{s}$. This is because as $K$ increases, the PAPR of the OFDM/TDM signal reduces, and the signal is less degraded in the HPA. It is seen from Figure 5 that as $K$ increases the required peak-power (i.e., IBO) of OFDM/TDM is reducing; for the average $\mathrm{BER}=10^{-4}$, IBO can be reduced by about $1.3,2.9$ and $5.1 \mathrm{~dB}$, compared to the conventional OFDM, when $K=4,16$, and 64, respectively, as shown in Figure 5. The worst performance is achieved with the conventional OFDM $(K=1)$ due to large PAPR.

\section{B. Power efficiency issue}

In this section, we discuss about the peak-power that is proportional to the PAPR of the transmitted signal. By definition, it can be shown that the theoretical PAPR of OFDM/TDM is proportional to the number of subcarriers $N_{m}\left(=N_{c} / K\right)$. The PAPR values (in decibels) of

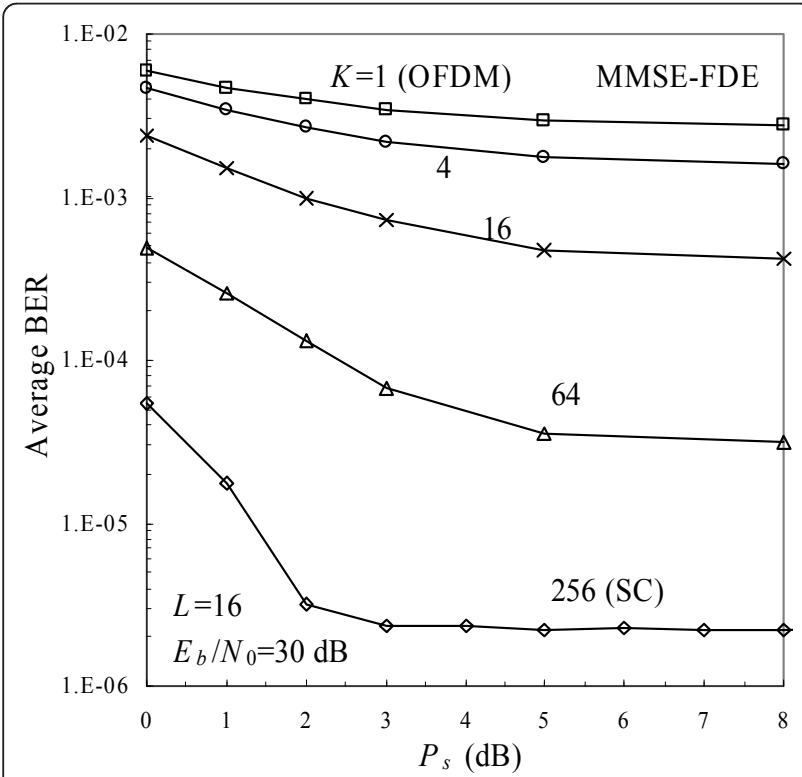

Figure 5 BER versus $P_{s}$
OFDM/TDM and conventional OFDM, which represent the required IBO for QPSK constellation are given in Table 1. It is seen from the table that the PAPR of OFDM is as large as $24 \mathrm{~dB}$, while, for OFDM/TDM with $K=4$ and 16 , the PAPR reduces to 18 and $12 \mathrm{~dB}$, respectively. Although the PAPR increases linearly with the number of subcarriers $N_{m}$, the probability that such a peak will occur decreases exponentially with $N_{m}$.

Figure 6 illustrates the theoretical and computer-simulated complementary cdf (ccdf) of PAPR for OFDM/ TDM as a function of $K$ when $N_{c}=256$. The theoretical ccdf of OFDM/TDM and the conventional OFDM are computed using (8). Also presented below are the computer simulation results for the OFDM/TDM signal transmission to confirm the validity of the theoretical analysis. Computer simulation results for ccdf of PAPR are obtained over 20 million OFDM/TDM frames. A fairly good agreement with theoretical and computersimulated results is seen, which confirms the validity of our PAPR analysis based on the Gaussian approximation of the OFDM/TDM signal. It can be seen from the figure that, as $K$ increases, the $\mathrm{PAPR}_{10 \%}$ level, by which the PAPR of OFDM/TDM exceeds with a probability of $10 \%$, is about $9,8,6.5$, and $3 \mathrm{~dB}$ for $K=1$ (OFDM), 4 , 16 , and 256 (SC), respectively.

We also consider the required peak transmit power because it is an important design parameter of transmit power amplifiers. For conventional OFDM transmission, high PAPR causes signal degradation due to nonlinear power amplification, and the BER performance degrades. Figure 7 illustrates the BER performance of the coded OFDM/TDM using MMSE-FDE as a function of the peak transmit power with $K$ as a parameter. We consider the PAPR $10 \%$ level, which the PAPR of OFDM/ TDM exceeds with a probability of $10 \%$. PAPR $10 \%$ are about 8.5, 7.2, and $5.7 \mathrm{~dB}$ for $K=1,16$, and 64, respectively. It is seen from the figure that for turbo code the conventional OFDM $(K=1)$ gives the worst performance due to the large PAPR. As $K$ increases the required peak-power (i.e., IBO) of OFDM/TDM is reducing; for the average BER $=10^{-4}$, IBO can be reduced by about 1.3, 2.9, and $5.1 \mathrm{~dB}$, compared to the conventional OFDM, when $K=4,16$ and 64, respectively, as shown in Figure 4. In the case of LDPC codes the performance improvement is slightly larger in comparison with turbo-coded performance. We note here that the

Table 1 PAPR comparison between OFDM/TDM and conventional OFDM

\begin{tabular}{lll}
\hline Parameters & $\boldsymbol{N}_{\boldsymbol{c}}=\mathbf{2 5 6}, \boldsymbol{N}_{\boldsymbol{m}}=\boldsymbol{N}_{\boldsymbol{c}} / \boldsymbol{K}$ & PAPR level (dB) \\
\hline Conventional OFDM & $K=1, N_{m}=256$ & 24.08 \\
\hline OFDM/TDM & $K=4(16), N_{m}=64(16)$ & $18.06(12.04)$ \\
\hline
\end{tabular}




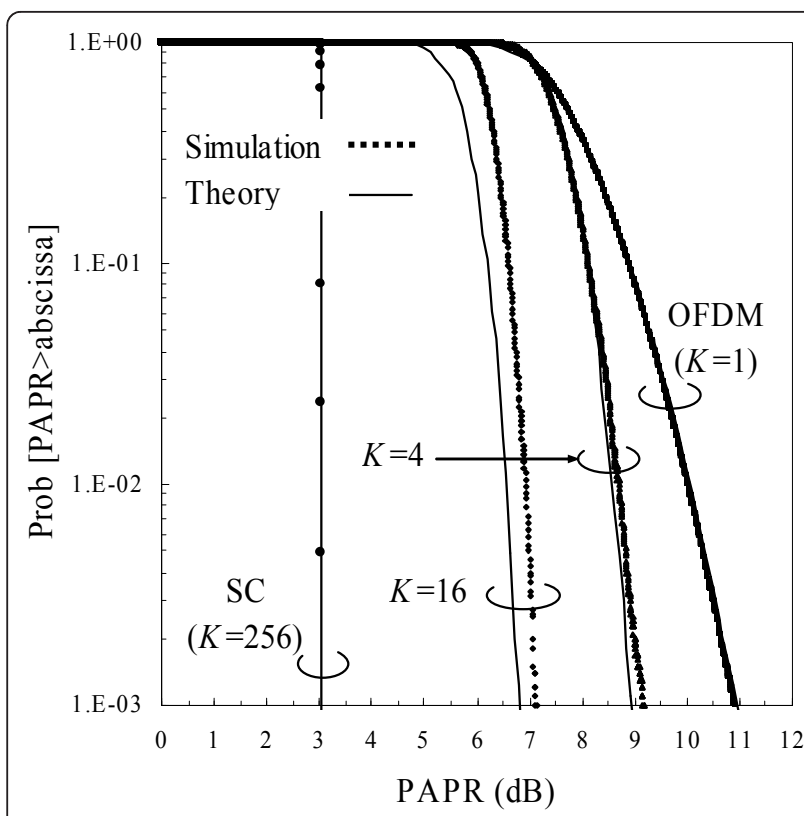

Figure 6 PAPR distribution of OFDM/TDM.

performance improvement presented above is paid with lower spectral efficiency as presented in the next section.

\section{Channel capacity issue}

The channel capacity in bps/Hz is illustrated in Figure 8 as a function of the amplifier's saturation power level $P_{s}$ normalized by the input signal power with $K$ as a parameter for $E_{b} / N_{0}=30 \mathrm{~dB}$ (for a low $E_{b} / N_{0}$ the achievable capacity is almost the same irrespective of $K$, and the capacity trade-off as a function of $K$ cannot be observed). The capacity of OFDM/TDM using MMSEFDE is illustrated in Figure 8 as a function $P_{s}$ for the average bit energy-to-AWGN power spectrum density ratio $E_{b} / N_{0}=30 \mathrm{~dB}$, where $E_{b} / N_{0}=0.5 \times\left(E_{s} / N_{0}\right) \times(1+$ $\left.N_{g} / N_{c}\right)$. The figure shows that for lower $P_{s}(<8 \mathrm{~dB})$, the performance of OFDM/TDM using MMSE-FDE with $K$ $=4,16$ and 64 outperforms the conventional OFDM $(K$ $=1)$, while the best capacity is achieved with SC-FDE $(K$ = 256) payed by the lower signal bandwidth occupancy. On the contrary, for higher $P_{s}(>8 \mathrm{~dB})$ the highest capacity is achieved with the conventional OFDM $(K=1)$, while the lowest is achieved with SC-FDE $(K=256)$.

\section{Channel code rate issue}

Here, the impact of different code rates on the BER performance with $K$ as a parameter is evaluated by computer simulation. Figure 9 illustrates the BER performance as a function of design parameter $K$ for both turbo- and LDPC channel-coding techniques. It can be seen from

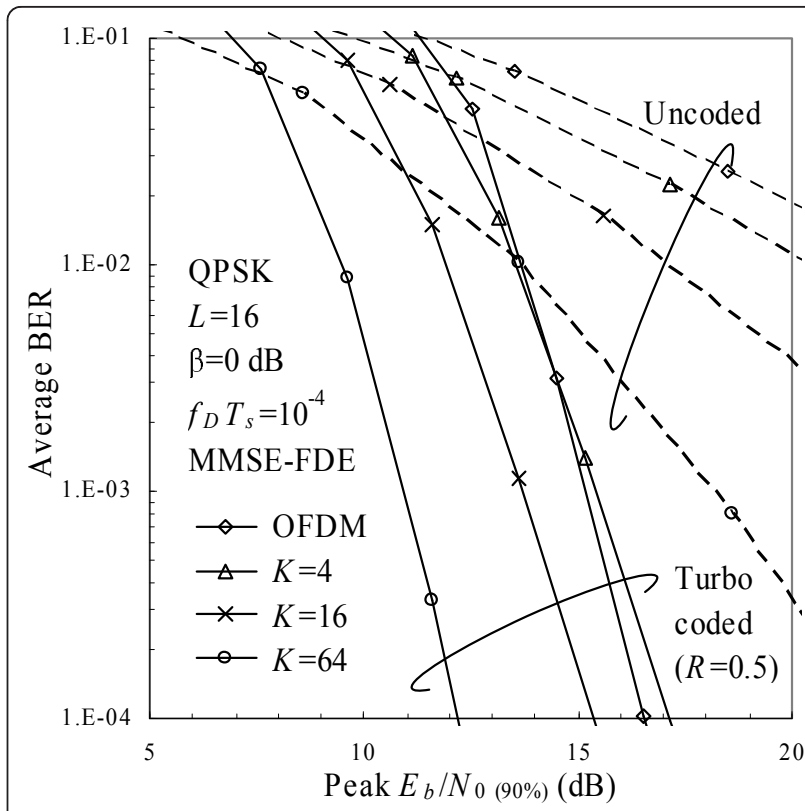

(a) Turbo coded

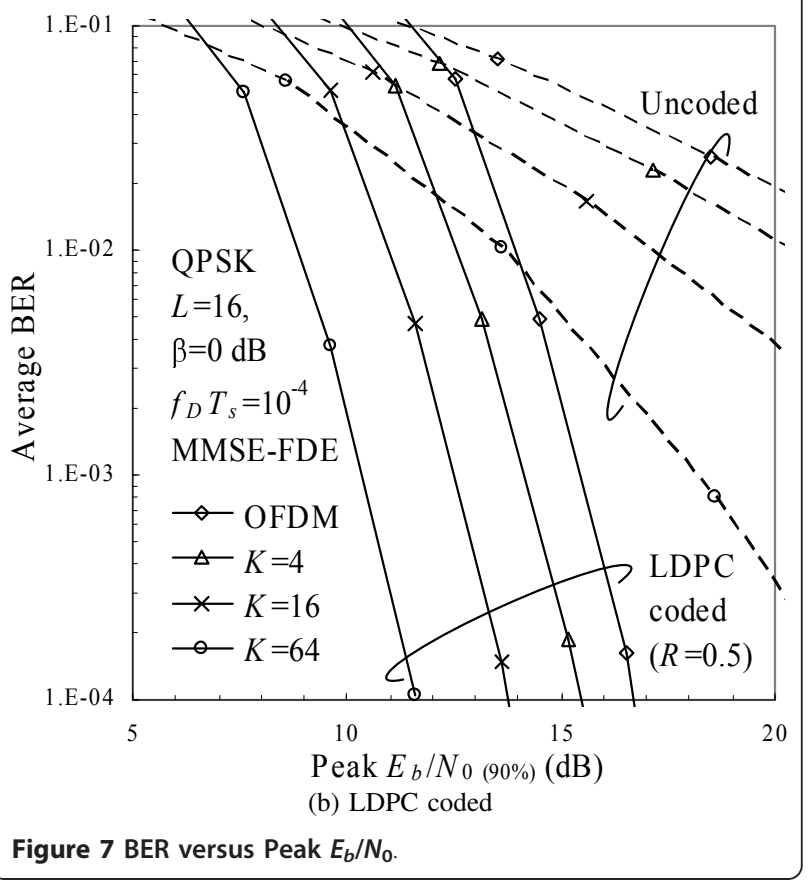

the figure that the impact of $K$ on the BER performance with different code rates is not high for both channel encoders. We note here that the impacts of different decoding strategies are not taken into consideration, and it are out of the scope of this study.

\section{E. The channel frequency-selectivity issue}

As said earlier, the performance improvement of OFDM/TDM is attributed to the frequency-diversity effect achieved by the MMSE-FDE. This suggests that 


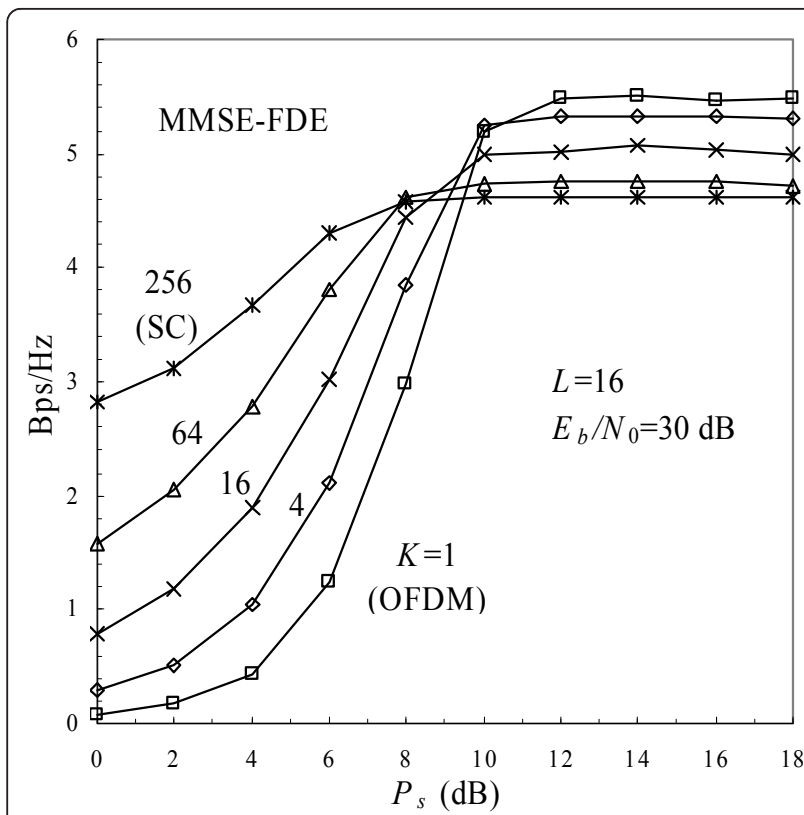

Figure 8 Impact of $P_{s}$ on capacity.

the BER performance depends on the channel frequency selectivity. The measure of the channel selectivity is the decay factor $\beta$ of the channel power-delay profile. The dependency of the achievable BER performance on $\beta$ is shown in Figure 10 for both turbo and LDPC encoders. As was expected, as $\beta$ becomes larger, the performance of OFDM/TDM with higher $K$ degrades for both encoders due to less frequency-diversity effect resulting from the weaker frequency selectivity. It can be also seen from the figure that in the case of LDPC channel encoder, the BER performance of OFDM/TDM is more stable in comparison with the performance of turbo channel encoder.

\section{F. Transmit signal bandwidth issue}

In this section, our focus is on the spectral efficiency of the OFDM/TDM and conventional OFDM. The PSD is computed over a sequence of 64,000 frames with $J=16$ oversampled OFDM/TDM waveform and averaged $10^{6}$ times. Figure 11 illustrates the PSD of OFDM/TDM ( $K=4$ and 16) and conventional OFDM $(K=1)$ with the amplifier's power saturation level $P_{s}=$ $4 \mathrm{~dB}$. It is seen from the figure that OFDM/TDM achieves a lower spectral efficiency in comparison with the conventional OFDM; the spectral efficiency decreases as $K$ increases. This is because OFDM/TDM signals have discontinuity in their waveforms within the OFDM/TDM frame and cause a higher-order spectral spreading. However, a better PSD of conventional OFDM is achieved at a cost of higher PAPR and BER, as discussed above.

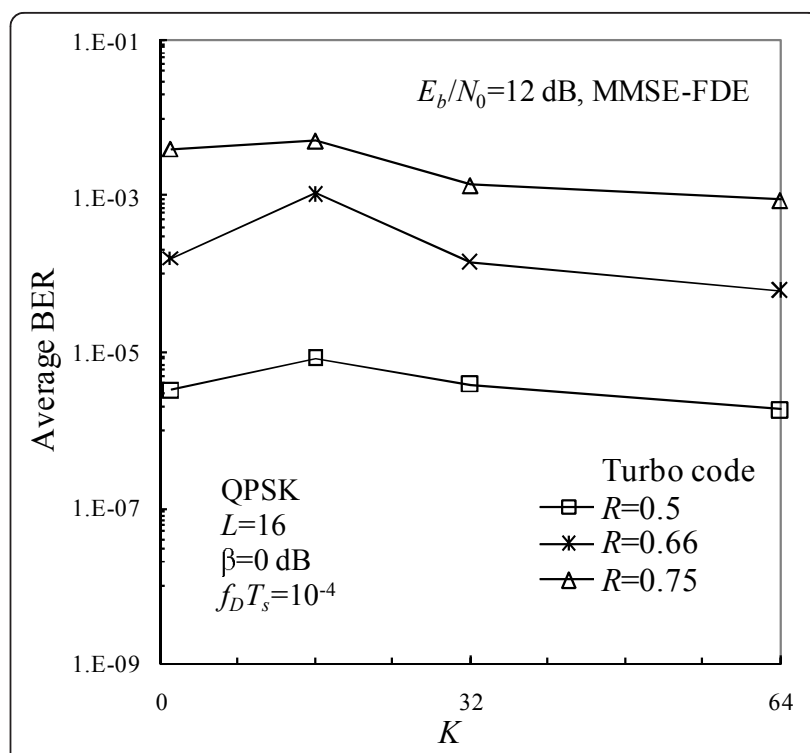

(a) Turbo coded

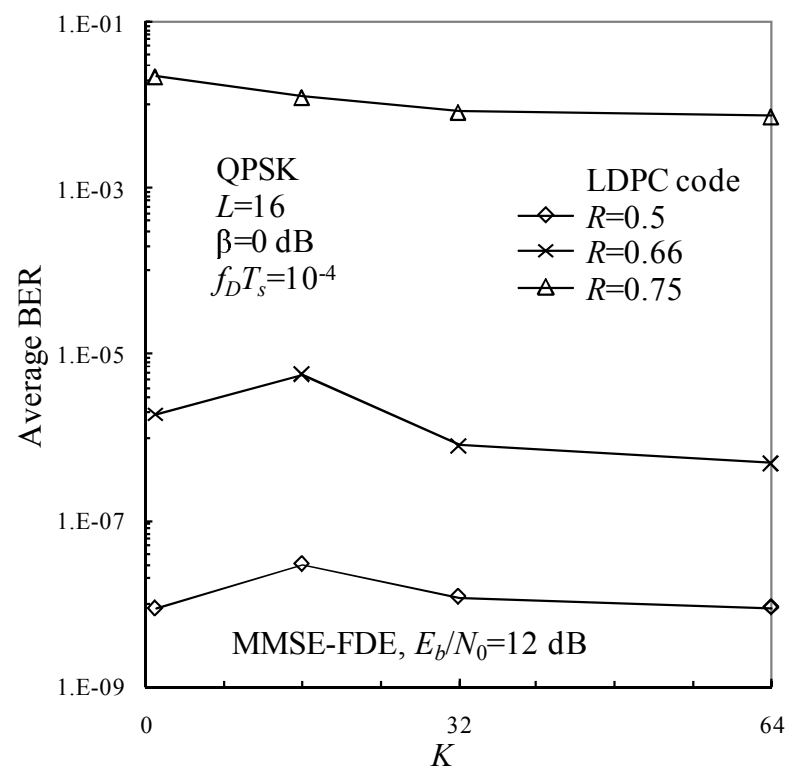

(b) LDPC coded

Figure 9 BER versus $K$.

\section{G. Complexity issue}

The computational complexity of OFDM/TDM has been evaluated in [20] by using the number of the required complex multiplications of IFFT/FFT operation as the comparison metric. It has been shown that the complexity of OFDM/TDM transmitter is lower than the complexity of its receiver, while the complexities of transmitter and receiver for the conventional OFDM are almost the same. On the other hand, the total (i.e., transmitter/receiver) complexity of OFDM/TDM is 


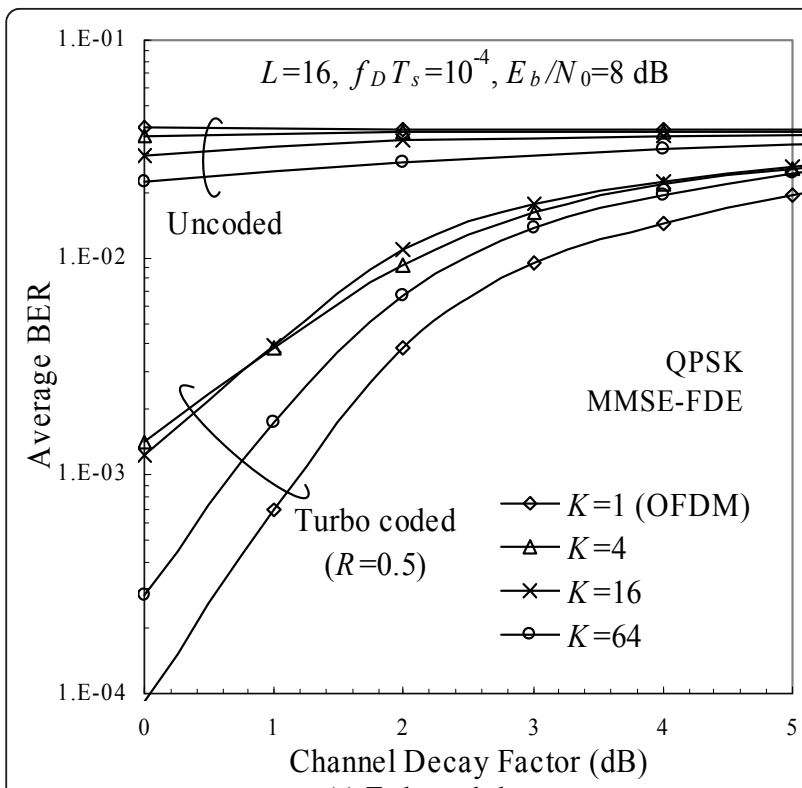

(a) Turbo coded

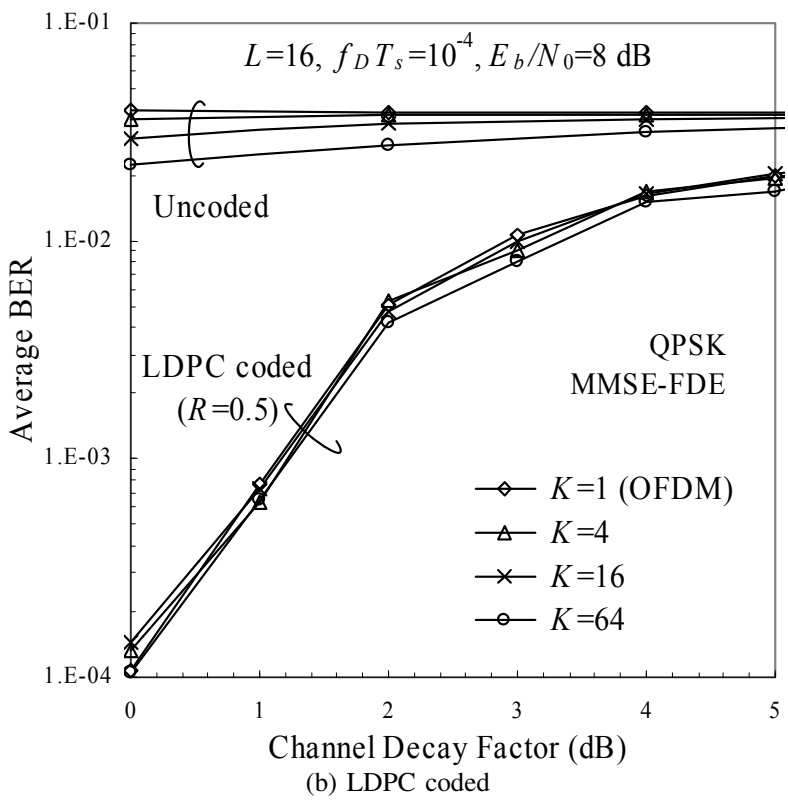

Figure 10 BER versus channel decay factor $\beta$.

larger in comparison with the complexity of the conventional OFDM [20].

\section{v. Conclusion}

In this article, we have analyzed and discussed a tradeoff between the peak-power reduction, the channel capacity, and the spectrum efficiency for OFDM/TDM using MMSE-FDE was presented. It was shown that the OFDM/TDM reduces the peak-transmit power (i.e., IBO) for the same BER, but with a slight increase in PSD in comparison with the conventional OFDM. It was also shown that OFDM/TDM using MMSE-FDE

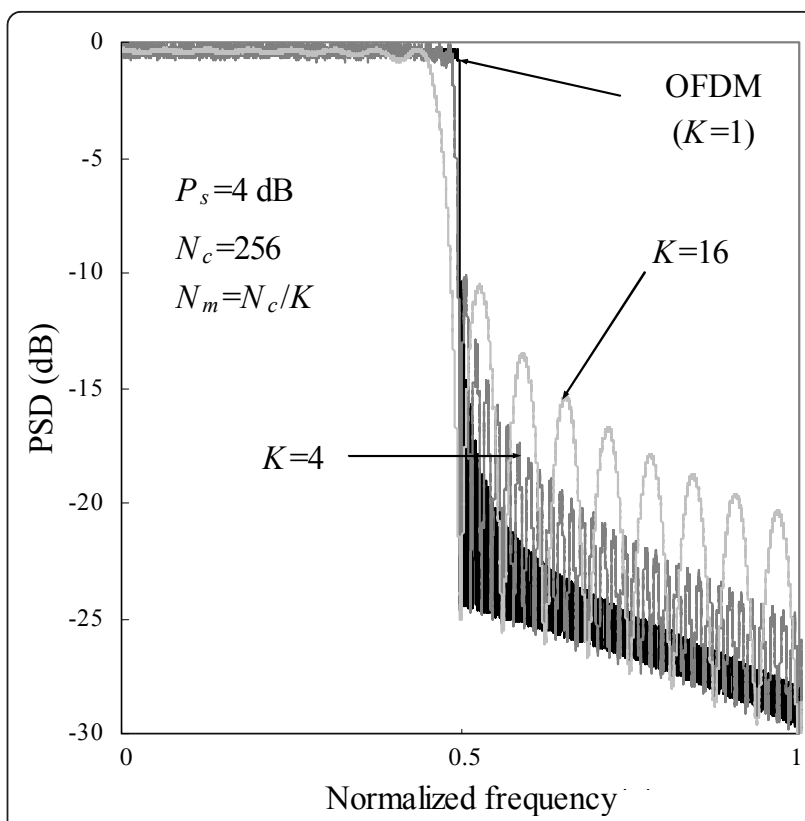

Figure 11 PSD performance

can be designed to achieve a higher capacity with a lower PAPR in comparison with the conventional OFDM in a nonlinear and frequency-selective fading channel. Hence, OFDM/TDM using MMSE-FDE provides flexibility in designing an OFDM-based systems.

\section{Acknowledgements}

This study was supported in part by 2010 KDDI Foundation Research Grant Program.

\section{Author details}

'Motive Division, Alcatel-Lucent Bell N.V., Antwerp, Belgium ${ }^{2}$ Graduate School of Engineering, Tohoku University, Sendai, Japan

\section{Competing interests}

The authors declare that they have no competing interests.

Received: 4 July 2011 Accepted: 2 December 2011

Published: 2 December 2011

\section{References}

1. JG Proakis, Digital communications, 3rd edn. (McGraw-Hill, New York, 1995)

2. S Hara, R Prasad, Multicarrier Techniques for $4 \mathrm{G}$ Mobile Communications (Artech House, Norwood, MA, 2003)

3. D Falconer, SL Ariyavisitakul, A Benyamin-Seeyar, Eidson B, Frequencydomain equalization for single-carrier broadband wireless systems. IEEE Commun Mag. 40, 58-66 (2002)

4. M Friese, On the degradation of OFDM signals due to peak-clipping in optimally predistorted power amplifiers, in Proceeding of IEEE GlobeCom, vol. 2. (Sydney, Australia, 1998), pp. 939-944

5. P Banelli, S Cacopardi, Theoretical analysis and performance of OFDM signals in nonlinear AWGN channels. IEEE Trans Commun. 48(3), 430-441 (2000)

6. D Dardari, V Tralli, A Vaccari, A theoretical characterization of nonlinear distortion effects in OFDM systems. IEEE Trans Commun. 48(10), 1755 (2000)

7. R O'Neill, LB Lopes, Envelope Variations and Spectral Splatter in Clipped Multicarrier Signals, in Proceedings of the 1995 IEEE International Symposium on Personal, Indoor and Mobile Radio Communications (PIMRC 1995), Toronto, Canada, pp. 71-75 (11-14 September 1995) 
8. AE Jones, TA Wilkinson, SK Barton, Block coding scheme for reduction of peak to mean envelope power ratio of multicarrier transmission scheme. Elect Lett. 30(22), 2098-2099 (1994)

9. BS Krongold, DL Jones, PAR reduction in OFDM via active constellation extension. IEEE Trans Broadcasting 49(3), 258-268 (2003)

10. SH Muller, JB Huber, OFDM with reduced peak-to-average power ratio by optimum combination of partial transmit sequences. Elect Lett. 33(5), 368-369 (1997)

11. RW Bauml, RFH Fisher, JB Huber, Reducing the peak-to-average power ratio of multicarrier modulation by selected mapping. Elect Lett. 32(22), 2056-2057 (1996)

12. P Van Eetvelt, G Wade, M Tomlinson, Peak to average power reduction for OFDM schemes by selective scrambling. Elect Lett. 32(21), 1963-1964 (1996)

13. Z Wang, X Ma, G Giannakis, OFDM or single-carrier block transmission. IEEE Trans Commun. 52(3), 380-394 (2004)

14. H Ochiai, H Imai, Performance analysis of deliberately clipped OFDM signals. IEEE Trans Commun. 50(1), 89-101 (2002)

15. CV Sinn, J Gotze, M Haardt, Common architectures for TD-CDMA and OFDM based mobile radio systems without the necessity of a cyclic prefix, in Proceedings of the MS-SS Workshop, DLR, Oberpfaffenhofen, Germany (24-25 September 2001)

16. H Gacanin, S Takaoka, F Adachi, OFDM combined with TDM using frequency-domain equalization. J Commun Netw. 9(1), 34-42 (2007)

17. H Gacanin, F Adachi, PAPR advantage of amplitude clipped OFDM/TDM. IEICE Trans Commun. E91-B(3), 931-934 (2008)

18. C Berrou, A Glavieux, P Thitimajshima, Near optimum error correcting coding and decoding: Turbo codes. IEEE Trans Commun. 44(10), 1261-1271 (1996)

19. T Wadayama, A coded modulation scheme based on low density parity check codes. IEICE Trans Commun. E81-B(10), 1-5 (2001)

20. H Gacanin, S Takaoka, F Adachi, Bit error rate analysis of OFDM/TDM with frequency-domain equalization. IEICE Trans Commun. E89-B(2), 509-517 (2006)

doi:10.1186/1687-1499-2011-193

Cite this article as: Gacanin and Adachi: On transmission performance of OFDM-based schemes using MMSE-FDE in a frequency-selective fading channel. EURASIP Journal on Wireless Communications and Networking 2011 2011:193.

\section{Submit your manuscript to a SpringerOpen ${ }^{\mathcal{O}}$ journal and benefit from:}

- Convenient online submission

- Rigorous peer review

- Immediate publication on acceptance

- Open access: articles freely available online

- High visibility within the field

- Retaining the copyright to your article

Submit your next manuscript at $\gg$ springeropen.com 